\title{
ATIVIDADE ANTIMICROBIANA DE CIMENTOS ENDODÔNTICOS
}

\section{ANTIMICROBIAL ACTIVITY OF ROOT-CANAL SEALERS}

\author{
Ana Carolina Mascarenhas Oliveira* \\ Cristiane Duque**
}

\begin{abstract}
RESUMO
A atividade antimicrobiana é uma propriedade fundamental dos cimentos endodônticos devido à permanência de microrganismos residuais no canal radicular, mesmo após o preparo químico-mecânico e o emprego do curativo de demora. O objetivo deste trabalho é realizar uma revisão de literatura a respeito das propriedades antimicrobianas de alguns dos principais cimentos endodônticos. Embora haja controvérsias em relação a essa propriedade, possivelmente devido a diferenças nas metodologias empregadas nos estudos, pode-se concluir que os cimentos com melhor atividade antimicrobiana foram (em ordem crescente): Endofill, Ketac Endo, Sealapex, AH Plus, Endo CPM Sealer, Sealer 26 e Epiphany. Activ GP ainda necessita de pesquisas científicas que avaliem essa propriedade.
\end{abstract}

DESCRITORES: Produtos com ação antimicrobiana • Cimentos dentários • Obturação do canal radicular

\section{ABSTRACT}

The antimicrobial activity is a fundamental property of root-canal sealers due to persistence of residual microorganisms in the root canal system, even after the chemo-mechanical preparation and using of intracanal dressing. The aim of this study is to review the literature about the antimicrobial properties of some of the main root-canal sealers. Although there is controversy regarding this property, probably due to differences in the methodologies used in the studies, it was concluded that the sealers with the best antimicrobial activity were (in ascending order): Endofill, Ketac Endo, Sealapex, AH Plus, Endo CPM Sealer, Sealer 26 and Epiphany. Activ GP still needs scientific research to evaluate this property.

DESCRIPTORS: Products with antimicrobial action • Dental cements • Root canal obturation 


\section{N T R O D UÇÃ O}

A obturação do canal radicular visa o preenchimento do espaço anteriormente ocupado pela polpa dental. É realizada após o adequado preparo químico-mecânico e a desinfecção do sistema de canais radiculares. O selamento obtido através da obturação é fundamental para o restabelecimento e manutenção da saúde apical e periapical, pois evita a infiltração microbiana, seja por via apical ou coronária, entre as paredes do canal radicular e o material obturador, diminuindo a possibilidade de insucesso do tratamento endodôntico ${ }^{1}$.

Para tanto, além de um preparo do canal radicular adequadamente realizado, o material e a técnica empregados na obturação são fundamentais. Os materiais obturadores devem possuir adequadas propriedades físico-químicas e biológicas, como bom escoamento, viscosidade e aderência, biocompatibilidade aos tecidos periapicais e atividade antimicrobia$n^{2}$.

O material sólido mais comumente utilizado na obturação dos canais radiculares é a guta-percha. Para garantir o preenchimento do espaço entre os cones de guta-percha e as irregularidades do canal radicular são utilizados os cimentos obturadores ${ }^{3}$. Há atualmente no mercado diversos cimentos que são agrupados de acordo com sua constituição química em: cimentos à base de óxido de zinco e eugenol como o Endofill; à base de ionômero de vidro, como o Activ GP e Ketac Endo; à base de Mineral Trióxido Agregado (MTA), como o Endo CPM Sealer; aqueles que contêm hidróxido de cálcio, como o Sealapex; e os cimentos à base de resina, como o AH Plus, Sealer 26 e Epiphany ${ }^{2}$. O objetivo deste trabalho foi realizar uma revisão de literatura a respeito das propriedades antimicrobianas de alguns dos principais cimentos obturadores endodônticos.

\section{REVISÃO DE LITERATURA}

\section{Propriedades dos cimentos obtura- dores}

De acordo $\mathrm{com}^{2}$, quanto às propriedades físico-químicas, o cimento obtura- dor deve possuir adequada capacidade de escoamento, viscosidade e aderência, promovendo um bom selamento do canal radicular. Deve apresentar-se, ao final da manipulação, com consistência plástica, permitindo um bom tempo de trabalho até tornar-se sólido. Deve ser radiopaco, não manchar o elemento dental, não ser solubilizado no canal radicular, além de ser passível de esterilização e de fácil remoção do canal radicular.

Quanto às propriedades biológicas, os cimentos obturadores endodônticos devem ser biocompatíveis aos tecidos apicais/periapicais, promoverem a estimulação osteogênica e, principalmente, apresentarem atividade antimicrobiana. Essa propriedade é considerada fundamental devido à possibilidade de permanência de microrganismos nos túbulos dentinários e ramificações do canal principal, mesmo após o preparo químico-mecânico e o emprego do curativo de demora. Assim, o cimento obturador deve eliminar a microbiota remanescente e permitir o adequado selamento de canalículos dentinários e ramificações do sistema de canais radiculares ${ }^{2}$.

Devido à importância da desinfecção dos canais radiculares, a literatura endodôntica tem se preocupado com a análise das propriedades antimicrobianas dos materiais endodônticos. Entretanto, ainda há dúvidas em relação à ação de alguns materiais sobre microrganismos comumente encontrados no sistema de canais radiculares. A seguir, são citados alguns dos principais cimentos obturadores e suas principais propriedades, enfatizando-se a atividade antimicrobiana de cada um deles.

\section{Cimentos obturadores \\ AH Plus}

$\mathrm{O}$ AH Plus (Dentsply/De Trey, Konstanz, Alemanha) é um cimento obturador que apresenta em sua composição resina epóxica, óleo de silicona e tungstênio. Não possui prata e recentemente houve uma alteração em seu radiopacificador que passou de óxido de bismuto para óxido de zircônio. Estudos têm demonstrado que esse cimento possui adequadas propriedades físico-químicas ${ }^{4,5,6}$. Apresenta baixa solubilidade e adequada estabilida-
MASCARENHAS - OLIVEIRA AC DUQUE C

ATIVIDADE ANTIMICROBIANA DE CIMENTOS ENDODONTICOS

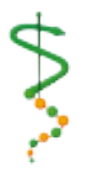

REV, ODONTOL.

UNIV, CID, SÃo

PAULO

$2013 ; 25(1): 58$

67 , JAN-ABR 
MASCARENHAS -

- OLIVEIRA AC

DUQUE C

AT IVIDADE

AN T IMI CROB I ANA

DE CIMENTOS

ENDODONTICOS

\section{0}

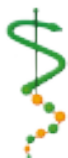

REV, ODONTOL

UNIV, CID, SÃO

PAULO

$2013 ; 25(1): 58$

67 , JAN-ABR
I SSN 1983-5183

de dimensional, o que lhe confere maior imbricação nos túbulos dentinários e menor infiltração marginal ${ }^{4,5}$. É considerado de fácil manipulação por apresentar-se em duas pastas. Sua composição resinosa e elevado tempo de polimerização promovem melhor escoamento e penetração nas microirregularidades.

Esse cimento apresenta atividade antimicrobiana de moderada a satisfatória contra diversos microrganismos, como Streptococcus mutans, Streptococcus sanguinis, Escherichia coli, Staphylococcus aureus, Porphyromonas gingivalis, Porphyromonas endodontalis, Fusobacterium nucleatum, Prevotella intermedia, Actinomyces naeslundii, Prevotella nigrescens, Streptococcus mitis, Streptococcus bovis, Lactobacillus casei e Candida albicans ${ }^{7,8,9,}$ e também contra $E$. faecalis $^{10,11}$. Carvalho-Junior et al. ${ }^{6}$ (2007) afirmam que as características do AH Plus podem ser atribuídas à sua matriz resinosa que é resistente à solubilidade, o que leva à redução das falhas na obturação, evitanto a penetração de $E$. faecalis.

\section{Epiphany}

O sistema Epiphany/Resilon (Pentron Clinical Technologies, LLC, Wallingford, CT, USA) é composto por um cimento hidrofílico de cura dual à base de uma mistura de UDMA (uretano de metacrilato), PEGDMA (polietileno dimetacrilato), EBPADMA (etoxilato bisfenol A dimetacrilato) e BISGMA (bisfenol A metacrilato glicídio), um primer autocondicionante e cones sintéticos à base de polímero de poliéster, vidro bioativo e dimetacrilato. Nesse sistema de obturação, o ResiIon substitui a guta-percha e apresenta adesão ao Epiphany e este às paredes do canal radicular, formando um monoblo$\mathrm{CO}^{12}$, que confere estabilidade e resistência à obturação. O Epiphany SE (self-etch) apresenta resinas e metacrilatos em sua composição ${ }^{13}$, sendo proposto em substituição ao Epiphany para ser utilizado sem a necessidade prévia do primer, devido à resistência dos profissionais em adicionar a etapa de condicionamento ao protocolo de obturação.

Apesar de alguns trabalhos terem mostrado que o cimento Epiphany apresentou selamento satisfatório e consequente resistência à infiltração contra bactérias como E. faecalis, quando comparado ao $\mathrm{AH}$ Plus e guta-percha, não houve diferença, sugerindo não haver vantagem aparente com seu uso ${ }^{14,15}$. Além disso, algumas avaliações in vitro mostraram que Epiphany apresenta pouca ${ }^{16}$, ou nenhuma atividade antimicrobiana ${ }^{17,18}$.

\section{Sealer $\mathbf{2 6}$}

O Sealer 26 (Dentsply, Rio de Janeiro, RJ, Brasil) é um cimento obturador que apresenta em sua fórmula hidróxido de cálcio e resina epóxica ${ }^{7}$, assim como a encontrada no cimento AH 26. A resina epóxica presente no Sealer 26 é responsável pela adaptação da obturação às paredes dentinárias, bem como sua menor capacidade de dissolução e, consequentemente, melhor estabilidade dimensional, propiciando resistência à infiltração microbiana. Carvalho-Junior et al. ${ }^{19}$ (2003) observaram que o Sealer 26 apresenta-se de acordo com as normas da especificação $n^{\circ} 57$ da ADA (American Dental Association) para as propriedades de desintegração, solubilidade e alteração dimensional.

Quanto à ação antimicrobiana do Sealer 26, ainda há muita controvérsia na literatura, possivelmente por diferenças nas metodologias empregadas. Esse cimento apresentou moderada ação antimicrobiana, sendo tal ação maior quando comparada ao $\mathrm{AH}$ Plus $^{20}$, e menor quando comparado ao Endofill ${ }^{21}$. Observaram ${ }^{22}$ que esse cimento demonstrou moderada atividade contra E. coli, E. faecalis, Bacillus cereus e $S$. aureus. Contudo ${ }^{7}$ observaram ausência de ação antimicrobiana desse cimento contra E. faecalis. Apesar disso, o cimento tem demonstrado boa capacidade seladora como material obturador de canais radiculares $^{23}$, e como material retro-obturador em cirurgias parendodônticas ${ }^{7}$. Siqueira Junior et al..$^{24}$ (1999) observaram que $37 \%$ dos espécimes obturados com Sealer 26 e $80 \%$ dos obturados com Sealapex apresentaram contaminação após teste de infiltração por saliva, demonstrando que o Sealer 26 apresenta adequado selamento às paredes dos canais radiculares.

\section{Sealapex}

O cimento obturador à base de hidróxido de cálcio Sealapex (Sybron Endo, Orange, CA, USA) apresenta a capacida- 
de de induzir a formação de selamento biológico apical por tecido mineralizado após obturação do canal radicular ${ }^{25}$. Diferentes trabalhos têm reportado a ação antimicrobiana desse cimento ${ }^{26,27}$. Yasuda et al. ${ }^{28}$ (2008) observaram atividade antimicrobiana do Sealapex contra $S$. aureus, E. faecalis, C. albicans, S. mutans e S. sanguinis. Outros estudos ${ }^{29,30}$, também observaram atividade antimicrobiana do Sealapex contra $E$. faecalis.

Quanto à resistência à infiltração microbiana, o Sealapex tem demonstrado resultados favoráveis ${ }^{31}$. Cortez ${ }^{32}$ (2005) observou que obturações com esse cimento, expostas ao meio bucal, foram resistentes à infiltração coronária por até 180 dias. Devido à baixa radiopacidade desse cimento, o radiopacificador em sua fórmula foi substituído de sulfato de bário para trióxido de bismuto. Essa troca pode acarretar modificações em suas propriedades físico-químicas e biológicas, sendo importante a avaliação antimicrobiana dessa nova formulação.

\section{Activ G P}

O desenvolvimento de cimentos à base de ionômero de vidro tem sido fundamentado na adesão que estes podem apresentar à dentina do canal radicular. O sistema de obturação Activ GP (Brasseler USA, Dental Instrumentation, USA) é composto por um cimento de ionômero de vidro e cones de guta-percha impregnados e cobertos por $2 \mu \mathrm{m}$ de ionômero de vidro para aumentar sua adesão ao cimento. Assim como Resilon/Epiphany, a adesão desse cimento às paredes do canal radicular é referida como um monobloco ${ }^{33}$. Entretanto $^{34}$ afirmam que a cobertura de cimento sobre a superfície dos cones do sistema Activ GP não é homogênea, o que contribui para uma adesão desfavorável.

São escassas as informações acerca das propriedades antimicrobianas do cimento Activ GP. Em relação à sua capacidade de resistência à infiltração microbiana, alguns trabalhos já podem ser encontrados. Fransen et al. ${ }^{33}$ (2008), avaliando a resistência à infiltração por $E$. faecalis do Activ GP, AH Plus e Epiphany, verificaram que não houve diferença significante entre esses cimentos. Monticelli et al. ${ }^{35}$ (2007) observaram que obturações com Activ GP resultaram em níveis de infiltração por $S$. mutans significantemente maiores quando comparadas ao AH Plus.

\section{Ketac Endo}

O Ketac Endo (ESPE Dental-Medizin, Alemanha) é um cimento à base de ionômero de vidro com apresentação em cápsulas de plástico (Ketac-Endo Aplicap) que são pressionadas durante dois segundos para liberação do líquido, sendo então levadas ao amalgamador a 4.300 vibrações por minuto, durante dez segundos, para homogeneizar o pó e o líquido. Após essa fase, a cápsula é adaptada em outro dispositivo que a pressiona para promover a saída do cimento pelo bico de plástico dispensador, inserindo-o no canal radicu$\operatorname{lar}^{36}$.

Esse cimento possui facilidade de manipulação e inserção ${ }^{37}$, biocompatibilidade tecidual $^{38}$, formação de tags nos canalículos dentinários ${ }^{39}$, adesão às paredes dentinárias do canal radicular e aos cones de guta-percha ${ }^{39}$, adequado selamento coronário ${ }^{40}$, resistência à fratura radicular em dentes tratados endodonticamente ${ }^{37}$, e constante liberação de flúor ${ }^{37,39}$, o que pode interferir no processo de formação de cárie radicular.

O Ketac Endo apresenta atividade antimicrobina satisfatória. Abdulkader et al. ${ }^{41}$ (1996), analisando a ação de cinco cimentos sobre $P$. gingivalis, Capnocytophaga ochracea e Peptostreptococcus micros, observaram que o Roth (Roth Drug Co., Chicago, IL, USA) obteve maior halo de inibição, seguido do Ketac Endo, Tubliseal (SybronEndo, Orange, CA, USA), Apexit (Ivoclar Vivadent Inc, Schaan, Liechtenstein) e Sealapex. Cobankara et al. ${ }^{10}$ (2004) avaliaram a atividade antibacteriana de diferentes cimentos sobre $E$. faecalis. Ketac Endo, Sultan (Sultan Chemists, USA) e AH Plus obtiveram resultados semelhantes no teste de contato direto com os microrganismos e maior inibição bacteriana em relação ao Sealapex e Roeko Seal (Roeko Dental Products, Langenau, Germany). Outros estudos ${ }^{10,16,42}$ mostraram que o Ketac Endo possui atividade antimicrobiana relevante e ação sobre $E$. faecalis, enquanto ${ }^{11}$ observaram que o Ketac Endo apresenta moderada efetividade em eliminar E. faecalis presente em túbu-
MASCARENHAS - OLIVEIRA AC DUQUE C

ATIVIDADE ANTIMICROBIANA DE CIMENTOS ENDODONTICOS 61

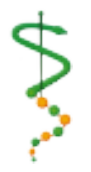

REV, ODONTOL.

UNIV. CID, SÃo PAULO

$2013 ; 25(1): 58-$ 67 , JAN-ABR 
MASCARENHAS -

- OLIVEIRA AC

DUQUE C

AT IVIDADE

AN TIMICROB I ANA

DE CIMENTOS

ENDODONTICOS

62

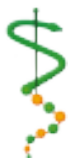

REV, ODONTOL.

UNIV, C I D . SÃO

PAULO

2013; 25(1): 58

67 , JAN-ABR
I SSN 1983-5183

los dentinários.

Em relação à sua resistência à infiltração microbiana ${ }^{4}$, avaliando dentes infectados com E. faecalis, observaram que após 30 dias não houve diferença entre os cimentos Ketac Endo e AH Plus, mas o Apexit apresentou infiltração significante. Após 60 dias, não houve diferença significante entre Ketac Endo e Apexit, porém o AH Plus apresentou melhores resultados.

\section{Endo CPM Sealer}

O MTA vem sendo amplamente preconizado em endodontia para o tratamento de perfurações, capeamentos pulpares, pulpotomia e como material obturador retrógrado. Sua composição básica é a do cimento Portland, cujos componentes principais são silicato tricálcico, silicato dicálcico, aluminato tricálcico, ferro aluminato tetracálcico e sulfato de cálcio hidratado. Esse material possui o mecanismo de ação biológica semelhante ao hidróxido de cálcio ${ }^{43}$.

Em 2003, foi lançado na Argentina o cimento Endo CPM Sealer (Egeo S.R.L. Bajo licencia MTM S.A., Buenos Aires, Argentina) para obturação de canais radiculares, sendo sua formulação semelhante ao $\mathrm{MTA}^{44}$. Segundo ${ }^{45}$, o CPM é um cimento Portland modificado, de cor branca, com características especiais de plasticidade, aderência, tamanho das partículas, $\mathrm{pH}$, tolerância biológica, histocompatibilidade, estimulação osteogênica e escoamento. Bramante et al. ${ }^{46}$ (2006) citaram as vantagens do Endo CPM Sealer em relação aos compostos derivados do óxido de zinco e eugenol, como seu excelente selamento marginal que impede a migração bacteriana e dos fluidos tissulares para o interior do canal e também o tamanho adequado das partículas do pó, o que lhe confere boa aderência às paredes do canal radicular. Vasconcelos ${ }^{47}$ (2006) verificou que o Endo CPM Sealer apresentou pH alcalino e foi capaz de liberar íons cálcio, porém, com tendência de redução dessa liberação em períodos mais longos, apresentando valores intermediários de tempo de presa em relação aos demais cimentos avaliados e com média de solubilidade de acordo com as normas de especificação da ADA.

Existe pouca informação quanto à atividade antimicrobiana do Endo CPM Sealer, entretanto, devido à semelhança entre as formulações do MTA e do CPM, espera-se semelhança também entre suas propriedades. O óxido de cálcio presente na composição desses materiais possui papel fundamental em sua atividade antimicrobiana. Quando essa substância é misturada à água forma-se hidróxido de cálcio, o que modifica os níveis de $\mathrm{pH}$ pela dissociação de íons cálcio e hidroxila ${ }^{48}$, elevando o pH inicial do MTA de 10,2 para $12,5^{49}$, e levando à morte de microrganismos, mesmo os resistentes como E. faecalis, que não suportam um $\mathrm{pH}$ tão alcalino ${ }^{50}$.

A atividade antimicrobiana do MTA foi avaliada por $^{49}$, que observaram sua eficácia contra bactérias facultativas. Asgary e Kamrani $^{27}$ (2008) avaliaram a atividade antibacteriana do MTA cinza e branco, hidróxido de cálcio $(\mathrm{HC})$, cimento Portland (CP) e um novo cimento (NEC) diante de Pseudomonas aeruginosa, E. faecalis, $S$. aureus, E. coli e uma mistura dessas bactérias. Não houve diferença significativa entre NEC e HC, e também entre MTA e CP. No entanto, houve uma significativa diferença entre HC e NEC, em comparação com MTA e CP.

Tanomaru et al. ${ }^{51}$ (2008) avaliaram a atividade antimicrobiana de diferentes cimentos contra Micrococcus luteus, $S$. aureus, $P$. aeruginosa, $C$. albicans e $E$. faecalis. Sealapex, Sealer 26, Endo CPM Sealer e MTA branco apresentaram atividade antimicrobiana contra todos os microrganismos testados. Intrafill (SS White, Rio de Janeiro, RJ, Brasil) não apresentou atividade antimicrobiana somente contra $P$. aeruginosa. Quanto à resistência à infiltração ${ }^{5}$, observou que AH Plus e CPM foram semeIhantes e permitiram pouca penetração de E. faecalis nos túbulos. Medeiros ${ }^{52}$ (2009) verificou que os cimentos MTA Branco (Angelus Ind. Com. Ltda, Londrina, PR, Brasil), MBPC (cimento experimental) e CPM apresentaram resistência à infiltração bacteriana.Endofill

O Endofill (Dentsply Ind. Com. Ltda, Petrópolis, RJ, Brasil) é um cimento à base de óxido de zinco e eugenol que apresenta adequadas propriedades físico-químicas, porém, quando extravasado, provoca uma 
resposta inflamatória nos tecidos periapicais $^{2}$. Essa inflamação ocorre devido à liberação de eugenol que é uma substância citotóxica; porém, esse componente confere relevante capacidade antimicrobiana ao cimento. Queiroz et al. ${ }^{30}$ (2009) avaliaram a atividade antimicrobiana do óxido de zinco e eugenol (OZE), pasta Calen espessada com óxido de zinco (Calen/ OZ), Sealapex e Endorez (Ultradent, South Jordan, UT, USA) perante Kocuria rhizophila, E. faecalis, S. mutans, E. coli e $S$. aureus. Em ordem decrescente de eficácia tem-se OZE, Calen/OZ, Sealapex e Endorez. Leonardi et al. ${ }^{22}$ (2009) avaliaram a ação antimicrobiana do Endofill, Sealer 26, AHPlus e Acroseal (Septodont, Saint Maur des Fosses, Cedex, France) pelo método de difusão em ágar contra $E$. coli, $E$. faecalis, B. cereus e $S$. aureus. Endofill evidenciou alto poder antimicrobiano principalmente contra E. coli.

Em relação à resistência à infiltração ${ }^{31}$, avaliaram obturações com Endofill e Sealapex. Quando a cultura de E. faecalis foi renovada, ocorreu turvação em 17 espécimes obturados com Endofill e em 7 obturados com Sealapex. Pinheiro et al. ${ }^{15}$ (2009) observaram que AH Plus e Endofill apresentaram resistência à infiltração por E. faecalis estatisticamente equivalentes entre si.

\section{CONCLUSÃO}

Embora haja controvérsias em relação à propriedade antimicrobiana de alguns cimentos endodônticos, possivelmente devido a diferenças nas metodologias empregadas nos estudos, pode-se concluir que os cimentos com melhor atividade antimicrobiana foram (em ordem crescente): Endofill, Ketac Endo, Sealapex, AH Plus, Endo CPM Sealer, Sealer 26 e Epiphany. Activ GP ainda necessita de pesquisas científicas que avaliem essa propriedade.
MASCARENHAS -

- OLIVEIRA AC

DUQUE C

ATIVIDADE

ANTIMICROBIANA

DE CIMENTOS

ENDODONTICOS

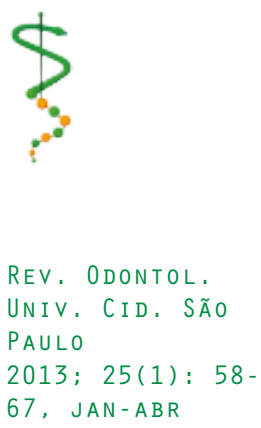


MASCARENHAS-

- OLIVEIRA AC

DUQUE C

ATI IVIDADE ANT IMI CROB I ANA

DE CIMENTOS

ENDODONTICOS
REV. ODONTOL.

UNIV. CID, SÃO PAULO $2013 ; 25(1): 58-$ $67, \mathrm{JAN}-\mathrm{ABR}$

\section{REFERÊNCIAS}

1. Siqueira Junior F, Rocas I, Alves F, Campos L. Periradicular status related to the quality of coronal restorations and root canal fillings in a Brazilian population. Oral Surg Oral Med Oral Pathol Oral Radiol Endod 2005 Sep;100(3):369-74.

2. Leonardo M. Endodontia: tratamento de canais radiculares, princípios técnicos e biológicos. São Paulo: Artes Médicas; 2005.

3. Soares I, Goldberg F. Obturação do canal radicular. In: Soares I, Goldberg F, editors. Endodontia: técnica e fundamentos. 3 ed. Porto Alegre: Artmed; 2001 p. 41-55.

4. Timpawat S, Amornchat C, Trisuwan WR. Bacterial coronal leakage after obturation with three root canal sealers. J Endod 2001 Jan;27(1):36-9.

5. Bortolini M. Avaliação de cimentos endodônticos a penetração intratubular e microinfiltração por Enterococcus faecalis [Mestrado]. Taubaté, SP: Universidade de Taubaté; 2006.

6. Carvalho-Junior JR, Correr-Sobrinho L, Correr AB, Sinhoreti MA, Consani S, Sousa-Neto MD. Solubility and dimensional change after setting of root canal sealers: a proposal for smaller dimensions of test samples. J Endod 2007 Sep;33(9):1110-6.

7. Siqueira Junior F, Favieri A, Gahyva S, Moraes S, Lima K, Lopes H. Antimicrobial activity and flow rate of newer and established root canal sealers. J Endod 2000 May;26(5):274-7.

8. Lai CC, Huang FM, Yang HW, Chan Y, Huang MS, Chou MY, et al. Antimicrobial activity of four root canal sealers against endodontic pathogens. Clin Oral Investig 2001 Dec;5(4):236-9.

9. Gomes BP, Pedroso JA, Jacinto RC, Vianna ME, Ferraz CC, Zaia AA, et al. In vitro evaluation of the antimicrobial activity of five root canal sealers. Braz Dent J 2004 15(1):30-5.

10. Cobankara FK, Altinoz HC, Ergani O, Kav K, Belli S. In vitro antibacterial activities of root-canal sealers by using two different methods. J Endod 2004 Jan;30(1):57-60.

11. Saleh IM, Ruyter IE, Haapasalo M, Orstavik D. Survival of Enterococcus faecalis in infected dentinal tubules after root canal filling with different root canal sealers in vitro. Int Endod J 2004 Mar;37(3):193-8.

12. Teixeira FB, Teixeira EC, Thompson JY, Trope M. Fracture resistance of roots endodontically treated with a new resin filling material. J Am Dent Assoc 2004 May;135(5):646-52.

13. Ungor M, Onay EO, Orucoglu H. Push-out bond strengths: the Epiphany-Resilon endodontic obturation system compared with different pairings of Epiphany, Resilon, AH Plus and gutta-percha. Int Endod J 2006 Aug;39(8):643-7.

14. Baumgartner G, Zehnder M, Paque F. Enterococcus faecalis type strain leakage through root canals filled with Gutta-Percha/AH plus or Resilon/Epiphany. J Endod 2007 Jan;33(1):45-7.

15. Pinheiro CR, Guinesi AS, de Camargo EJ, Pizzolitto AC, Filho IB. Bacterial leakage evaluation of root canals filled with different endodontic sealers. Oral Surg Oral Med Oral Pathol Oral Radiol Endod 2009 Dec;108(6):e56-60.

16. Neelakantan P, Subbarao CV. An analysis of the antimicrobial activity of ten root canal sealers--a duration based in vitro evaluation. J Clin Pediatr Dent 2008 Winter;33(2):117-22. 
17. Slutzky-Goldberg I, Slutzky H, Solomonov M, Moshonov J, Weiss El, Matalon S. Antibacterial properties of four endodontic sealers. J Endod 2008 Jun;34(6):735-8.

18. Pinheiro CR, Guinesi AS, Pizzolitto AC, Bonetti-Filho I. In vitro antimicrobial activity of Acroseal, Polifil and Epiphany against Enterococcus faecalis. Braz Dent J 2009 20(2):107-11.

19. Carvalho-Junior JR, Guimaraes LF, Correr-Sobrinho L, Pecora JD, Sousa-Neto MD. Evaluation of solubility, disintegration, and dimensional alterations of a glass ionomer root canal sealer. Braz Dent J 2003 14(2):114-8.

20. Kopper P, Rosa R, Figueiredo J, Pereira C, Tartarotti E, Filippini H. Avaliação in vitro da atividade antimicrobiana de três cimentos endodônticos. Rev Odonto Ciência 2007 22(56):106-11.

21. Signoretti F, Gomes B, JA P, Teixeira F, Vianna M, Jacinto R, et al. Avaliação in vitro da atividade antimicrobiana de cinco cimentos endodônticos. $19^{a}$ Reunião Anual da SBPqO; São Paulo: Universidade de São Paulo; 2003. p. 61.

22. Leonardi D, Battisti J, Klimiont D, Tomazinho P, Baratto Filho F, Haragushiku G, et al. Avaliação in vitro da ação antimicrobiana de alguns cimentos endodônticos. Rev Sul-Bras Odontol 2009 dez.;6(4):367-73.

23. Siqueira Junior J, Garcia Filho P. Avaliação in vitro das propriedades seladoras de três cimentos endodônticos à base de hidróxido de cálcio. Rev Odontol Bras 1994 51(1):37-40.

24. Siqueira Junior F, Rocas I, Lopes H, Uzeda M. Coronal leakage of two root canal sealers containing calcium hydroxide after exposure to human saliva. J Endod 1999 Jan;25(1):14-6.

25. Holland R, de Souza V. Ability of a new calcium hydroxide root canal filling material to induce hard tissue formation. J Endod 1985 Dec;11(12):535-43.

26. Leonardo MR, da Silva LA, Tanomaru Filho M, Bonifacio KC, Ito IY. In vitro evaluation of antimicrobial activity of sealers and pastes used in endodontics. J Endod 2000 Jul;26(7):391-4.

27. Asgary S, Kamrani FA. Antibacterial effects of five different root canal sealing materials. J Oral Sci 2008 Dec;50(4):469-74.

28. Yasuda Y, Kamaguchi A, Saito T. In vitro evaluation of the antimicrobial activity of a new resin-based endodontic sealer against endodontic pathogens. J Oral Sci 2008 Sep;50(3):309-13.

29. Mickel AK, Nguyen TH, Chogle S. Antimicrobial activity of endodontic sealers on Enterococcus faecalis. J Endod 2003 Apr;29(4):257-8.

30. Queiroz AM, Nelson-Filho P, Silva LA, Assed S, Silva RA, Ito IY. Antibacterial activity of root canal filling materials for primary teeth: zinc oxide and eugenol cement, Calen paste thickened with zinc oxide, Sealapex and EndoREZ. Braz Dent J 2009 20(4):290-6.

31. Silva K, Teixeira R, Lana M, Fróes J. Avaliação in vitro da infiltração bacteriana em canais radiculares obturados com dois diferentes cimentos. Anais da $19^{a}$ Reunião da Sociedade Brasileira de Pesquisa Odontológica - SBPqO; Águas de Lindóia,SP: SBPqO; 2002. p. 132.

32. Cortez D. Estudo in vivo da infiltração coronária em dentes de cães tratados endodonticamente protegido por diferentes materiais [Doutorado]. Piracicaba, SP: Universidade Estadual de Campinas; 2005. 
MASCARENHAS -

- OLIVEIRA AC

DUQUE C

ATI IVIDADE

ANTIMICROBI ANA

DE CIMENTOS

ENDODONTICOS

33. Fransen JN, He J, Glickman GN, Rios A, Shulman JD, Honeyman A. Comparative assessment of ActiV GP/glass ionomer sealer, Resilon/Epiphany, and gutta-percha/ AH plus obturation: a bacterial leakage study. J Endod 2008 Jun;34(6):725-7.

34. Monticelli F, Sword J, Martin RL, Schuster GS, Weller RN, Ferrari M, et al. Sealing properties of two contemporary single-cone obturation systems. Int Endod J 2007 May;40(5):374-85.

35. Monticelli F, Sadek FT, Schuster GS, Volkmann KR, Looney SW, Ferrari M, et al. Efficacy of two contemporary single-cone filling techniques in preventing bacterial leakage. J Endod 2007 Mar;33(3):310-3.

36. Vale M. Avaliação em microscopias óptica e eletrônica de varredura da adaptação de três cimentos endodônticos à dentina radicular submetida à ação prévia do laser de Er: YAG, EDTA e solução salina fisiológica [Doutorado]. Bauru, SP: Universidade de São Paulo; 2001.

37. Ray H, Seltzer S. A new glass ionomer root canal sealer. JEndod1991 Dec;1 7(12):598603.

38. Zmener O, Dominguez FV. Tissue response to a glass ionomer used as an endodontic cement. A preliminary study in dogs. Oral Surg Oral Med Oral Pathol 1983 Aug; 56(2):198-205.

39. Saunders EM, Saunders WP, Rashid MY. The effect of post space preparation on the apical seal of root fillings using chemically adhesive materials. Int Endod J 1991 Mar;24(2):51-7.

40. Saunders WP, Saunders EM. The effect of smear layer upon the coronal leakage of gutta-percha fillings and a glass ionomer sealer. Int Endod 1992 Sep;25(5):245-9.

41. Abdulkader A, Duguid R, Saunders EM. The antimicrobial activity of endodontic sealers to anaerobic bacteria. Int Endod J 1996 Jul;29(4):280-3.

42. Shalhav M, Fuss Z, Weiss El. In vitro antibacterial activity of a glass ionomer endodontic sealer. J Endod 1997 Oct;23(10):616-9.

43. Holland R, de Souza V, Nery MJ, Faraco Junior IM, Bernabe PF, Otoboni Filho JA, et al. Reaction of rat connective tissue to implanted dentin tube filled with mineral trioxide aggregate, Portland cement or calcium hydroxide. Braz Dent J 2001 12(1):38.

44. Ferreira M, Kairalla E, Hoshina R, Lage-Marques J. Análise de espectrometria de fluorescência de Raios- $X$ e Difratometria de Raios-X dos cimentos MTA, CPM e CPM Sealer. Anais da $22^{a}$ Reunião da Sociedade Brasileira de Pesquisa Odontológica SBPqO; Águas de Lindóia, SP2005. p. 165.

45. Castro G. Trióxidos minerales agregados - CPM e Endo CPM Sealer [Monografia]. Buenos Aires: Centro de Investigaciones Odontológicas; 2003.

46. Bramante C, Bramante A, Moraes I, Bernardineli N, Garcia R. CPM es MTA: nuevos materiales de uso en endodoncia - experiencias clinicas en el manejo de los materiales. Rev Fav Odontol 2006 17( ):7-10.

47. Vasconcelos B. Avaliação de algumas propriedades físico-químicas de cimentos retro-obturadores à base de agregado trióxido mineral e de um cimento epóxico experimental [Mestrado]. Bauru, SP: Universidade de São Paulo; 2006.

48. Duarte MA, Demarchi AC, Yamashita JC, Kuga MC, Fraga Sde C. pH and calcium ion release of 2 root-end filling materials. Oral Surg Oral Med Oral Pathol Oral Radiol Endod 2003 Mar;95(3):345-7. 
49. Torabinejad M, Hong CU, Pitt Ford TR, Kettering JD. Antibacterial effects of some root end filling materials. J Endod 1995 Aug;21 (8):403-6.

50. McHugh CP, Zhang P, Michalek S, Eleazer PD. pH required to kill Enterococcus faecalis in vitro. J Endod 2004 Apr;30(4):218-9.

51. Tanomaru JM, Tanomaru-Filho M, Hotta J, Watanabe E, Ito IY. Antimicrobial activity of endodontic sealers based on calcium hydroxide and MTA. Acta Odontol Latinoam 2008 21(2):147-51.

52. Medeiros P. Avaliação comparativa in vitro da capacidade seladora do cimento MBPc, CPM, MTA Angelus ${ }^{\circledR}$ branco e pasta Lysanda ${ }^{\circledR}$ à infiltração bacteriana em obturações retrógradas [Mestrado]. Bauru, SP: Universidade de São Paulo; 2009.

Recebido em:

Aceito em:

MASCARENHAS -

- OLIVEIRA AC

DUQUE C

ATIVIDADE

ANTIMICROBIANA

DE CIMENTOS

ENDODONTICOS

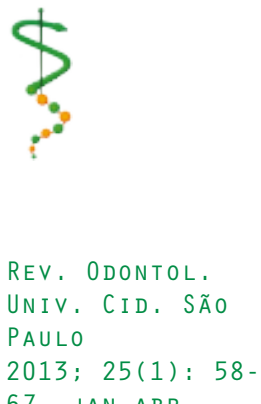

67 , JAN-ABR 